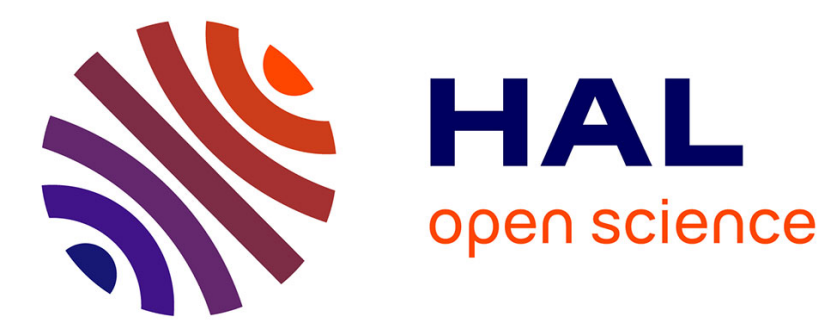

\title{
Application Performance Anomaly Detection with LSTM on Temporal Irregularities in Logs
}

\author{
Xavier Baril, Oihana Coustié, Josiane Mothe, Olivier Teste
}

\section{To cite this version:}

Xavier Baril, Oihana Coustié, Josiane Mothe, Olivier Teste. Application Performance Anomaly Detection with LSTM on Temporal Irregularities in Logs. CIKM '20: The 29th ACM International Conference on Information and Knowledge Management, Oct 2020, Virtual Event Ireland, Ireland. pp.1961-1964, 10.1145/3340531.3412157 . hal-03117074

\section{HAL Id: hal-03117074 \\ https://hal.science/hal-03117074}

Submitted on 20 Jan 2021

HAL is a multi-disciplinary open access archive for the deposit and dissemination of scientific research documents, whether they are published or not. The documents may come from teaching and research institutions in France or abroad, or from public or private research centers.
L'archive ouverte pluridisciplinaire HAL, est destinée au dépôt et à la diffusion de documents scientifiques de niveau recherche, publiés ou non, émanant des établissements d'enseignement et de recherche français ou étrangers, des laboratoires publics ou privés. 


\section{Application Performance Anomaly Detection with LSTM on Temporal Irregularities in Logs}

\author{
Xavier Baril \\ Oihana Coustié \\ xavier.baril@airbus.com \\ oihana.coustie@irit.fr \\ Airbus \\ Toulouse, France
}

\author{
Josiane Mothe \\ Olivier Teste \\ josiane.mothe@irit.fr \\ olivier.teste@irit.fr \\ ESPE, UT2J, Univ. de Toulouse, IRIT, UMR5505 CNRS \\ Toulouse, France
}

\begin{abstract}
Performance anomalies are a core problem in modern information systems, that affects the execution of the hosted applications. The detection of these anomalies often relies on the analysis of the application execution logs. The current most effective approach is to detect samples that differ from a learnt nominal model. However, current methods often focus on detecting sequential anomalies in logs, neglecting the time elapsed between logs, which is a core component of the performance anomaly detection. In this paper, we develop a new model for performance anomaly detection that captures temporal deviations from the nominal model, by means of a sliding window data representation. This nominal model is trained by a Long Short-Term Memory neural network, which is appropriate to represent complex sequential dependencies. We assess the effectiveness of our model on both simulated and real datasets. We show that it is more robust to temporal variations than current state-of-the-art approaches, while remaining as effective.
\end{abstract}

\section{CCS CONCEPTS}

- Software and its engineering $\rightarrow$ Software testing and debugging; • Theory of computation $\rightarrow$ Semi-supervised learning; - Computing methodologies $\rightarrow$ Anomaly detection; Neural networks.

\section{KEYWORDS}

information system, event logs, anomaly detection

\section{ACM Reference Format:}

Xavier Baril, Oihana Coustié, Josiane Mothe, and Olivier Teste. 2020. Application Performance Anomaly Detection with LSTM on Temporal Irregularities in Logs. In Proceedings of the 29th ACM International Conference on Information and Knowledge Management (CIKM '20), October 19-23, 2020, Virtual Event, Ireland. ACM, New York, NY, USA, 4 pages. https://doi.org/10.1145/3340531.3412157

Permission to make digital or hard copies of all or part of this work for personal or classroom use is granted without fee provided that copies are not made or distributed for profit or commercial advantage and that copies bear this notice and the full citation on the first page. Copyrights for components of this work owned by others than ACM must be honored. Abstracting with credit is permitted. To copy otherwise, or republish, to post on servers or to redistribute to lists, requires prior specific permission and/or a fee. Request permissions from permissions@acm.org.

CIKM '20, October 19-23, 2020, Virtual Event, Ireland

(C) 2020 Association for Computing Machinery.

ACM ISBN 978-1-4503-6859-9/20/10 _\$15.00

https://doi.org/10.1145/3340531.3412157

\section{INTRODUCTION}

With the fast growing of their size and complexity, modern information technology systems contain numerous sources of potential faults and vulnerabilities [3, 6, 8]. The failure of their services can have significant consequences, ranging from degraded user's experience [11] to important financial losses [15]. The ability to accurately detect anomalies is therefore considered as a major issue [15]. Log analysis constitute a promising solution to system anomaly detection, since large-scale systems generate numerous execution logs $[6,11]$. A $\log$ is a semi-structured message tracing the system execution [8]. It contains valuable monitoring information, such as the timestamp (time of occurrence) or the content, an often unstructured free text. The event type of a log describes the type of information being logged in the content part [1]. The sequence_id of a log is an optional field, gathering logs from a same sequence.

A $\log$ anomaly is an unexpected behaviour of the log data, and can be paired to a system anomaly. For instance, a late appearance of logs in a sequence may indicate a performance anomaly corresponding to an abnormal temporal irregularity in a service response. Hence, log anomaly detection is recognized as an efficient mean to perform system anomaly detection [3]. Manually analyzing large and complex log datasets represents a cumbersome and error-prone task [3, 8], justifying the need for automated data-driven solutions.

Supervised methods are the first historically used to detect anomalies in logs $[5,9]$. They train binary classifiers, based on both normal and abnormal samples. Despite their encouraging results on balanced data, they suffer from the scarcity of abnormal samples in real datasets. In addition, they only detect anomalies matching the learnt abnormal class, preventing the detection of new anomalies. Unsupervised outlier detection $[10,14]$ overcome both the scarcity of anomalies, and the frequent unavailability of labels. These clustering techniques isolate the nominal samples in polluted datasets. However, they tend to simply detect samples that are significantly different from the majority regarding selected features [8].

Finally, novelty detection methods focus on detecting deviations from the nominal behaviour. Following a semi-supervised approach, a nominal behaviour model is learnt on anomaly-free data. New coming data are compared to this model and detected as anomalies if their behaviour does not match the nominal one. In these methods, logs are often represented as temporally-ordered sequences of event types. Fu et al. [7] train a Finite State Automaton to model these sequences as a unique workflow. Yet, the unique aspect of the model prevents the treatment of sequences that contain parallel tasks [6]. The DeepLog method [6] rather train a prediction task 
with a Long-Short Term Memory (LSTM) neural network, which efficiently represents complex sequential dependencies based on multiple previous logs. DeepLog is regarded as one of the most up-to-date and accurate reference.

Nonetheless, representing the logs as sequences of event types raises challenging issues. Firstly, the sequences creation requires the knowledge of the sequence_id, which is not always available. Secondly, this representation discards the time-frame separating logs. This information is however key to define performance anomalies. The performance anomaly detection field focuses on detecting periods of slowdown or unavailability of a system or a service [2], and ranges from the detection of deny-of-service attacks [6] to the performance monitoring of cloud infrastructures [12]. For these anomalies, the sequential representation of the logs is insufficient: the time elapsed between logs must be integrated to the model in order to detect temporal irregularities in logs' appearance.

This paper focuses on the detection of performance anomalies and presents a new novelty detection approach named NoTIL, Novelty detection based on Temporal Irregularities in Logs. Based on the idea of counting the event types over time with a sliding window, NoTIL takes the time elapsed between logs into account by capturing the frequency of logs' appearance. NoTIL takes advantage of the LSTM ability to model complex temporal dependencies and applies it to model temporal correlations and detect temporal irregularities. In the following, section 2 details our NoTIL method. Section 3 evaluates its efficiency and section 4 concludes the paper.

\section{NOTIL ANOMALY DETECTION METHOD}

As a novelty detection method, NoTIL models the nominal behaviour of logs and detects anomalies as violations of the trained model. Especially, NoTIL trains a forecasting model and detects the samples with low prediction results as anomalies. In the context of performance anomaly detection, the prediction tasks and the data representation should be chosen so as to take into account the time elapsed between logs. Indeed, a performance anomaly is manifested within the logs as an abnormally long time-frame separating two consecutive logs. Contrary to the state-of-the-art methods, NoTIL represents the logs with a counting time window of the event types, taking into account the time elapsed between logs. We use an LSTM network for the prediction model, since they can model complex temporal relations $[6,11]$. This section formalizes the problem of detecting performance anomalies before describing the data representation and the prediction model.

Notations. We use curly brackets $\left\{a_{1}, . ., a_{k}\right\}$ to designate a set of $k$ unique elements; $\left(a_{1} . . a_{k}\right)$ denotes a sequence and $\llbracket a . . b \rrbracket$ stands for the sequence of integers between $a$ and $b$ (included). $|E|$ is the number of elements of a set $E$ and $\mathcal{P}(E)$ is its power set. The floor function \lfloor\rfloor is defined by: $\forall x \in \mathbb{R},\lfloor x\rfloor=\max \{m \in \mathbb{Z} \mid m \leq x\}$. We note $\wedge$ the logical AND between conditions. Finally, for a random variable $X$ on an event space $\Omega, F_{X}$ is its cumulative distribution function. $\forall \alpha \in \llbracket 0 . .1 \rrbracket, X_{\alpha}$ is the $\alpha$-upper critical value of the probability distribution of $X$, define by $F_{X}\left(X_{\alpha}\right)=1-\alpha ; \forall x \in \Omega, x$ is significantly higher than $X$, noted $x \gg X$, if $x>X_{\alpha}$.

Problem statement. Let $L$ be a dataset of logs, occurring in a discrete time-frame $\llbracket 1 . . T \rrbracket, T \in \mathbb{N}^{+} . E$ is the set of possible event types in $L$. For a $\log \ell \in L$, we note $e_{\ell} \in E$ its event type and $t_{\ell} \in \llbracket 1 . . T \rrbracket$ its timestamp. For any $\ell \in L$ and $e \in E$, we define $\operatorname{next}_{e}(\ell) \in L$ as the next closest log of type $e$ : the first log of type $e$ that happens after $\ell$. We define $d_{e}(\ell)=t_{\text {next }}(\ell)-t_{\ell}$, the duration between $\ell$ and next $t_{e}(\ell)$. We assume that the nominal value of such a duration is a random variable $D_{e_{\ell}}$ following an unknown distribution. The actual duration $d_{e}(\ell)$ corresponds to a performance anomaly if $d_{e}(\ell) \gg D_{e_{\ell} e}$. We assume that anomalies concern instants $t \in \llbracket 1 . . T \rrbracket$ and we define a labelling strategy $f$ as a function indicating which instant(s) are labelled abnormal: $f: L \times E \rightarrow \mathcal{P}(\llbracket 1 . . T \rrbracket)$. The performance anomaly detection problem consists in retrieving the set of temporal anomalies in $L$ : $T_{L}^{A}=\left\{f(\ell, e) \mid \ell \in L, e \in E \wedge d_{e}(\ell) \gg D_{e_{\ell} e}\right\} \subset \llbracket 1 . . T \rrbracket$.

Data representation. We define a window size $w \in \mathbb{N}^{*}$ and a temporal window $W_{i}$, with a beginning time $t_{W_{i}}$ and an ending time $t_{W_{i}}+w$, with $t_{W_{i}}<t_{W_{i}}+w \leq T$. A $\log \ell$ belongs to a window $W_{i}$ iff $t_{W_{i}} \leq t_{\ell}<t_{W_{i}}+w$. We represent $W_{i}$ by a counting vector $C_{i} \in \mathbb{N}^{|E|}$ that counts, for each event type $e \in E$, the number of occurrences of $e$ in the window $W_{i}$. The $j^{\text {th }}$ element of $C_{i}$ is expressed as $C_{i}^{(j)}=\left|\left\{\ell \in L \mid t_{\ell} \in \llbracket t_{W_{i}} . t_{W_{i}}+w \rrbracket \wedge e_{\ell}=e_{j}\right\}\right|$. We opt for a sliding window mechanism, and define $s$ as the sliding offset and $N$ the number of sliding windows is $L$.

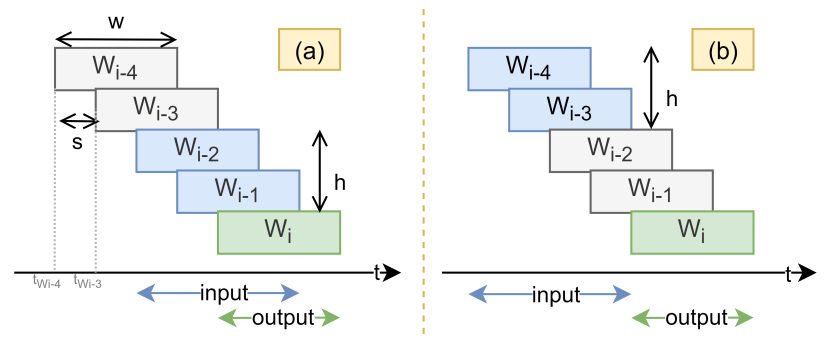

Figure 1: A set of $h=2$ input windows of size $w=6$ and shifted by $s=2$ (blue) and its associated output (green) in an overlapping scenario (a) and a non-overlapping one (b)

The prediction task. As in Deeplog, we train a model for the task of forecasting the next window. We define a hyper-parameter $h$, called look-back [6] so that $h$ consecutive windows are used to forecast the next window. Due to the sliding window mechanism, consecutive windows share common instants. To avoid the introduction of a bias between input and output - as depicted in the overlapping scenario in Figure 1(a) - we predict the output counting vector $C_{i}$ with the input matrix $M_{i}$ formed by the previous vectors $\left(C_{i-\alpha-h \ldots C_{i-\alpha}}\right)$, where $\alpha=\frac{w}{s}-1$ (Figure 1(b)). As aforementioned, we opt for a LSTM model to train the prediction task. We formalize its role as a function LSTM that associates to an input matrix $M_{i}$ a predicted output $\operatorname{LSTM}\left(M_{i}\right)$. This predicted output is then compared to the real output $C_{i}$ thanks to an error measure $\Delta$, a function defined as $\Delta: \mathbb{N}^{|E|} \times \mathbb{N}^{|E|} \rightarrow \mathbb{R}^{+}$.

Finally, a threshold $\tau$, learned during the validation, enables to split the windows into normal and abnormal subsets. The abnormal windows $W^{(A)}$ retrieved by NoTIL are defined as:

$$
W^{(A)}=\left\{W_{i} \mid \alpha+h \leq i<N, \Delta\left(C_{i}, \operatorname{LSTM}\left(M_{i}\right)\right)>\tau\right\}
$$




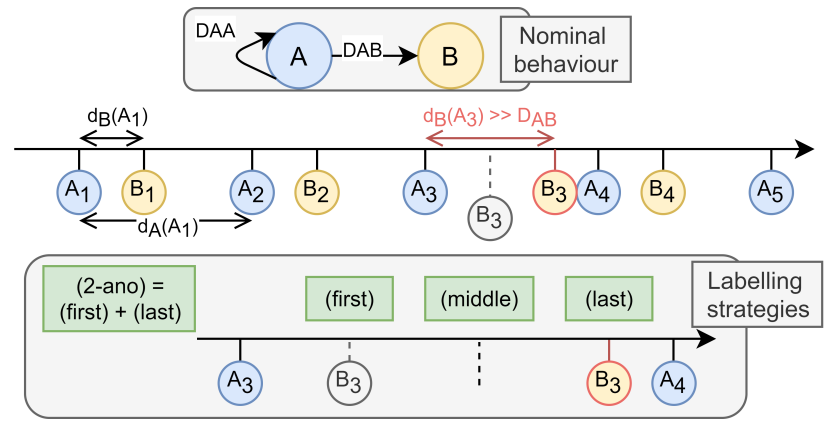

Figure 2: Generation scenario of Simu_binary. Top: nominal model. Center: temporal sequence with performance anomaly in $A_{3}-B_{3}$. Bottom: labelling strategies for the anomaly.

To compare this set to $T_{L}^{(A)}$, the set of actual temporal anomalies of $L$, we transpose this latter into a set of abnormal windows:

$$
W_{\text {real }}^{(A)}=\left\{W_{i} \mid \alpha+h \leq i<N \wedge \exists t \in T_{L}^{(A)}, t_{W_{i}} \leq t \leq t_{W_{i}}+w\right\}
$$

\section{EVALUATION}

We assess the accuracy of NoTIL on different scenarios and compare it with that of DeepLog [6]. We present the evaluation framework before commenting the experimental results.

Data presentation. We evaluate our proposition on both simulated and real-world datasets. We first generate an artificial dataset Simu_binary to study the behaviour of the methods in a minimal configuration. As depicted in Figure 2, Simu_binary contains 2 event types $A$ and $B . D_{A A}$ and $D_{A B}$ represent the nominal durations between consecutive logs of the corresponding event types. We inject performance anomalies in some transitions between $A$ and $B$ : in Figure 2, the transition between $A_{3}$ and $B_{3}$ is abnormal since $d_{B}\left(A_{3}\right) \gg D_{A B} . D_{A A}$ and $D_{A B}$ are Gaussian distributions with low variances (for steady scenarios). We generate several scenarios by changing the mean values. We then generate scenarios with higher variances to study the robustness of the methods to temporal irregularity. We also generate Simu_nevents, with new contextual event types that happen regularly, independently from one another and from the sequence of interest. This context matches better a real situation, where numerous types of logs are generated in parallel. In these artificial scenarios, we simulate a time-frame of $T=10000$ for each of the training, validation and testing sets, and inject $1 \%$ of anomalies in the validation and testing sets.

We finally experiment on a real dataset. Openstack logs monitors the creation and use of virtual machine (VM) instances. The authors of DeepLog generated and made available their own Openstack dataset with injected performance anomalies. The dataset contains 47 event types, among which 23 are implied in a sequence (a VM instance). 4 sequences contain temporal anomalies, in the same transition time, for a global rate of $0.7 \%$ of abnormal sequences.

State-of-the-art methods selection. We study the behaviour of DeepLog towards performance anomalies. DeepLog proposes two independent anomaly detectors: we note SDL its sequence anomaly detector, and PDL its performance anomaly detector. SDL only considers the sequence of event types, and is not specifically designed for performance anomalies. PDL processes each event type individually and analyses the time elapsed between consecutive logs. PDL is therefore eligible for performance anomaly detection: theoretically, it can catch temporal irregularities, but might struggle to detect abnormal correlations between logs of different event types. We also study the reference state-of-the-art unsupervised method noted PCA [14], in order to prove the superiority of the novelty approach.

Labelling strategy. For the simulated data, we propose different labelling strategies, defining the aforementioned function $f$ and graphically represented in the example of Figure 2. In these strategies, the abnormal instants $f\left(A_{3}, B\right)$ are:

- (first) the time when the log should have occurred, $t_{A_{3}}+D_{A B}$,

- (last) the time when the delayed log occurred, $t_{B_{3}}$,

- (2-ano) both,

- (middle) the middle instant between both, $\frac{t_{B_{3}}+t_{A_{3}}+D_{A B}}{2}$.

We study the impact of the labelling strategies on the results of the understudied methods in order to select a strategy that does not introduce any bias.

Implementation considerations. DeepLog and NoTIL are implemented with Pytorch framework and with the same hyperparameters provided in [13]. For our hyperparameters, we set $s=1$ to observe all the possible window combinations and experimentally determined the $w$ and $h$ values at $w=D_{A B}-d_{B}\left(A_{3}\right)$ (the difference between expected and observed duration) and $h=2 \times w$. PCA is not based on a prediction task, so the detection should be performed on a window size that covers both the input and the output of the other methods. Hence, we chose a window size of $w_{P C A}=w+h \times s$.
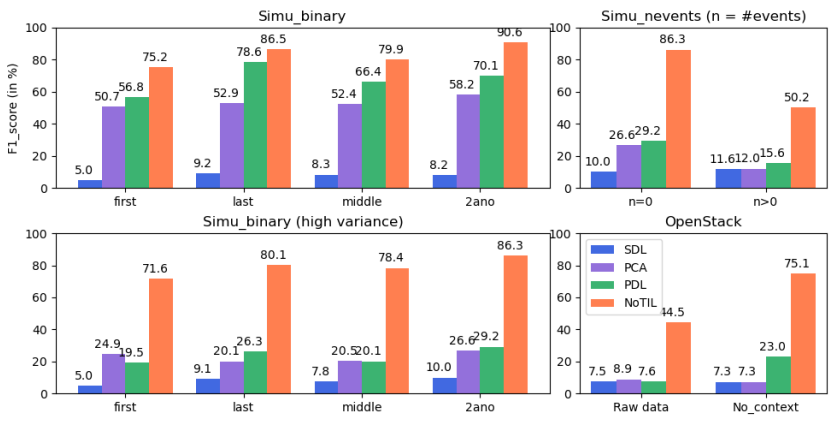

Figure 3: F1_scores of the understudied methods on simulated datasets and OpenStack.

Evaluation measures. Measuring the accuracy of an anomaly detector in our context consists in comparing the aforementioned sets $W_{\text {real }}^{(A)}$, the real abnormal windows and $W^{(A)}$, the predicted ones. The traditional F1-measure is not suitable for the performance anomaly context, since it does not tolerate any temporal delay between detected and real anomalies. Hence, we refine the definition of false positive and negative: for $q \in \mathbb{N}$, a real (resp. predicted) anomaly in $W_{i}$ is a false negative (resp. positive) if no detected (resp. real) anomaly is observable within the sequence 
$\left(W_{i-q} \ldots W_{i+q}\right)$, otherwise it is a true positive. We chose $q=\left\lfloor\frac{D_{A A}}{2}\right\rfloor$, in order to detect anomalies at a sequence-level temporal precision.

As most of the novelty detection methods, NoTIL learns a threshold to distinguish abnormal and normal samples. This threshold is learnt during a validation phase and is a lever to balance false positive and false negative. This step can be used to avoid detecting nominal outliers: novelty detection can detect anomalies that are more frequent than some nominal outliers, as long as they were not seen during the training phase.

Detection results. Figure 3 presents the F1-scores of the methods for the aforementioned datasets. For all the simulated data, we generated multiple scenarios - with different $D_{A A}$ and $D_{A B}$ values - and calculated the average score for each understudied method.

In the noise-free version of Simu_binary, both PDL and NoTIL present overall good results, with a slight overall advantage for NoTIL. Unsurprisingly, PDL benefits from the (last) labelling strategy, since it detects anomalies from the time elapsed since the previous log. $B_{3}$ and $B_{4}$ carry high anomaly scores since $B_{3}$ is abnormally far from $B_{2}$ and close to $B_{4}$. NoTIL also detects two abnormal instants, yet, these instants are aligned with the (2-ano) labels. The SDL proposal shows very disappointing results since the understudied anomalies have no signature at a sequence level, and are not observable if time is neglected. PCA method shows mitigate results, mainly due to the temporal imprecision necessary to encompass both the input and the output in the studied window.

The noisy version results highlight the limit of the PDL's representation of time. Since the occurrences of $A$ are not regular, neither are those of $B$. Hence, the method struggles in learning the nominal behaviour of the event type $B$, and fails to detect the actual anomalies. PCA also collapses: the multitude of nominal samples induces a lack of distinction between rare nominal profiles and anomalies. NoTIL is only slightly impacted by the irregularity of this scenario and still presents reliable results. Here, (2-ano) is unanimously the most advantageous labelling strategy: it is bias-free to select it for the next evaluations.

The experiments on Simu_nevents show an impact of the contextual framework on all methods: the already-low results of the state-of-the-art methods collapse, while NoTIL remains the only reliable solution, despite a fair decrease.

Finally, PDL presents very disappointing results in the raw OpenStack dataset (column Raw data): it tries to find a common forecasting error threshold, whatever the event type is. Since half of the event types are not implied in a sequence (contextual logs) and do not bear any signature of the anomalies, applying a threshold on these event types leads to numerous false positives. To confirm this hypothesis, we propose an alternative version of the dataset (column No_context), which only contains the 23 event types involved in a sequence execution. This process is not innocuous: it requires domain knowledge to identify the sequence_id and eliminates $61 \%$ of the logs. Yet, it is not sufficient: an event type occurs twice in each instance, hence why there are two nominal durations between consecutive occurrences: (a) a short one, between occurrences of the same instance, (b) a much longer one between distinct instances. PDL fails in learning this nominal behaviour and triggers many false positives. On the contrary, NoTIL results are enhanced by this simplification process. Our analysis of NoTIL results however highlighted some false positive cases, that seem reasonable. For instance, a rare event type appears at an unusually high frequency in the testing dataset which is detected as abnormal by NoTIL.

\section{CONCLUSION}

We present a new method to perform anomaly detection, while maintaining the quantitative aspect of time, using a count of event types over time. NoTIL efficiently detects temporal irregularities in logs' appearance, which can be the signatures of performance anomalies. Our evaluations show a much more robust behaviour towards nominal temporal fluctuations than current approaches such as DeepLog. As a future work, we plan to extend our evaluation to a larger set of anomaly types. We also aim at extending the anomaly detection to parameters anomalies: logs contain variable parts that can be converted into numerical time series. We will also experiment other deep learning algorithms, with a focus on attention mechanisms $[4,11]$ in order to enhance both the computational performance and the interpretability of the model.

\section{REFERENCES}

[1] Xavier Baril, Oihana Coustié, Josiane Mothe, and Olivier Teste. 2020. METING: A Robust Log Parser Based on Frequent n-Gram Mining. IEEE The International Conference on Web Services (ICWS) (2020).

[2] Stéphane Bonnevay, Jairo Cugliari, and Victoria Granger. 2019. Predictive Maintenance from Event Logs Using Wavelet-Based Features: An Industrial Application. In 14th Inter. Conf. on Soft Computing Models in Industrial and Environmental Applications (SOCO) (Advances in Intelligent Systems and Computing, Vol. 950). 132-141.

[3] Andrea Borghesi, Antonio Libri, Luca Benini, and Andrea Bartolini. 2019. Online Anomaly Detection in HPC Systems. In IEEE Inter. Conf. on Artificial Intelligence Circuits and Systems, AICAS. 229-233.

[4] Andy Brown, Aaron Tuor, Brian Hutchinson, and Nicole Nichols. 2018. Recurrent Neural Network Attention Mechanisms for Interpretable System Log Anomaly Detection. CoRR abs/1803.04967 (2018). arXiv:1803.04967

[5] Mike Y. Chen, Alice X. Zheng, Jim Lloyd, Michael I. Jordan, and Eric A. Brewer. 2004. Failure Diagnosis Using Decision Trees. In 1 st Inter. Conf. on Autonomic Computing (ICAC ). 36-43.

[6] Min Du, Feifei Li, Guineng Zheng, and Vivek Srikumar. 2017. DeepLog: Anomaly Detection and Diagnosis from System Logs through Deep Learning. In Proc. of the 2017 ACM SIGSAC Conference on Computer and Communications Security, CCS. 1285-1298.

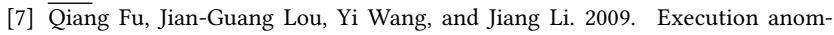
aly detection in distributed systems through unstructured log analysis. In 9th IEEE Inter. Conf. on data mining. 149-158.

[8] Shilin He, Jieming Zhu, Pinjia He, and Michael R. Lyu. 2016. Experience Report: System Log Analysis for Anomaly Detection. In 27th IEEE International Symposium on Software Reliability Engineering, ISSRE. 207-218.

[9] Yinglung Liang, Yanyong Zhang, Hui Xiong, and Ramendra K. Sahoo. 2007. Failure Prediction in IBM BlueGene/L Event Logs. In Proc. of the 7th IEEE Inter. Conf. on Data Mining (ICDM 2007). 583-588.

[10] Jian-Guang Lou, Qiang Fu, Shengqi Yang, Ye Xu, and Jiang Li. 2010. Mining Invariants from Console Logs for System Problem Detection. In USENIX Annual Technical Conference, 2010, Paul Barham and Timothy Roscoe (Eds.).

[11] Weibin Meng, Ying Liu, Yichen Zhu, Shenglin Zhang, Dan Pei, Yuqing Liu, Yihao Chen, Ruizhi Zhang, Shimin Tao, Pei Sun, and Rong Zhou. 2019. LogAnomaly: Unsupervised Detection of Sequential and Quantitative Anomalies in Unstructured Logs. In Proc. of the Twenty-Eighth International Joint Conference on Artificial Intelligence, IJCAI, Sarit Kraus (Ed.). 4739-4745.

[12] Yongmin Tan, Hiep Nguyen, Zhiming Shen, Xiaohui Gu, Chitra Venkatramani, and Deepak Rajan. 2012. PREPARE: Predictive Performance Anomaly Prevention for Virtualized Cloud Systems. In IEEE 32nd Inter. Conf. on Distributed Computing Systems. 285-294.

[13] Yifan Wu. 2018. DeepLog. https://github.com/wuyifan18/DeepLog.

[14] Wei Xu, Ling Huang, Armando Fox, David Patterson, and Michael Jordan. 2009. Largescale system problem detection by mining console logs. (2009).

[15] Ke Zhang, Jianwu Xu, Martin Renqiang Min, Guofei Jiang, Konstantinos Pelechrinis, and Hui Zhang. 2016. Automated IT system failure prediction: A deep learning approach. In IEEE Inter. Conf. on Big Data,. 1291-1300. 